\title{
Blood Pressure Screening in the Dental Office
}

\author{
Tiffany Blair, MPH, Susan Lawson, MHR, Heather Porter, \\ MPA, CHES
}

Ann Fam Med 2017;15:278. https://doi.org/10.1370/afm.2073.

\section{THE INNOVATION}

Integrating oral health and primary care has long been a goal for many health care organizations, including Federally Qualified Health Centers. The Ohio Association of Community Health Centers (OACHC) has recently built upon this principle through an initiative that makes blood pressure screening part of the intake process for oral health patients in member health centers-a step that makes good sense, given that many patients see a dentist more frequently than a physician.

\section{WHO \& WHERE}

$\mathrm{OACHC}$ is a membership organization that supports Ohio's community health centers, of which 33 offered on-site dental care at 81 sites when the blood pressure screening program began (Supplemental Appendix 1). In partnership with the Ohio Dental Association, and with funding assistance from the Ohio Dental Association Foundation, those 33 community health centers started checking the blood pressure of patients at every dental visit. (Since the beginning of the program in July, 2016, numbers have grown; now 36 of 49 community health centers offer on-site dental care at 83 sites.)

\section{HOW}

As part of this initiative, OACHC purchased automatic blood pressure monitors for each of the 81 dental sites, outfitting all of their 410 dental operatories with the equipment. OACHC also trained dental staff to use the machines and record the results. Furthermore, the association distributed a comprehensive policy and procedure for the screening (Supplemental Appendix 2), a patient education brochure (Supplemental Appendix 3), and a guide intended to help standardize the pro-

Conflicts of interest: autbors report none.

\section{CORRESPONDING AUTHOR}

Heather Porter, MPA, CHES

Ohio Association of Community Health Centers

2109 Stella Ct.

Columbus, $\mathrm{OH} 43215$

hporter@ohiochc.org cess (Supplemental Appendix 4). It turns out to be easy to take patients' blood pressure in the dental chair while educating them on risks and referring them to their physician if appropriate. OACHC held introductory webinars and continues to hold open calls for the health centers to ask questions about the procedures and collaborate on adapting their electronic dental records to collect and report the blood pressure data.

Preliminary data from the first reporting quarter (7/1/169/30/16) showed that 21,154 patients were screened during dental appointments, with 275 dental operatories (67\%) reporting. Other sites are working with their electronic dental record to provide reports in the future. Of the 23,479 screenings performed, 3,735 (15.9\%) identified elevated pressures (>140/90). Practices use a pocket reference card to guide them in responding to readings of various levels (Supplemental Appendix 3).

\section{LEARNING}

This program has facilitated communication and collaboration between the quality improvement staff of community health centers and oral health professionals and led to the addition of dental data to quality committee reports, the creation of user groups for electronic dental records and data sharing, and the improvement of data collection in community health centers. The sharing of unblinded data and quarterly publication of dashboard reports has generated some healthy competition amongst the dental teams and enhanced collaboration and networking between the community health centers.

Giving each community health center the appropriate tools and training has been critical to the success of this initiative. This a step forward in OACHC's goal to fully integrate oral health with primary care. It builds on an early childhood oral health program designed to incorporate oral health screenings, fluoride varnish applications, referrals, and caregiver oral health education and anticipatory guidance into well-child visits. Once we have all community dental centers incorporating blood pressure screenings into their daily routines, we can continue to guide them on initiatives that require the oral health and primary care teams to work together to achieve optimal total patient care.

Key words: primary care; dental health services; ambulatory blood pressure monitoring; community health centers, quality improvement

Author affiliations, supplemental appendixes, acknowledgments, and a note about funding support are available at http://www. AnnFamMed.org/content/15/3/278/suppI/DC1/. 